\title{
Investigating dense interstellar environments in the X-rays
}

\section{S.T. Zeegers}

SRON, Netherlands Institute for Space Research, Sorbonnelaan, 2, 3584 CA, Utrecht, The Netherlands

E-mail: S.t.zeegersdsron.np

\section{E. Costantini}

SRON, Netherlands Institute for Space Research, Sorbonnelaan, 2, 3584 CA, Utrecht, The Netherlands

E-mail: e.cestantiniesron.n

\section{C.P. de Vries}

SRON, Netherlands Institute for Space Research, Sorbonnelaan, 2, 3584 CA, Utrecht, The Netherlands

E-mail: c.p.de.vries.dsron.n]

\section{A.G.G.M. Tielens}

Leiden Observatory, Leiden University, PO Box 9513, 2300 RA, Leiden, The Netherlands E-mail: Eielensdstrw.leidenuniv.n]

\begin{abstract}
We present newly acquired laboratory data in the X-ray band of several silicate compounds, taken at the Soleil synchrotron facility in Paris. We focus in particular on the features caused by the silicon and magnesium absorption. Magnesium and silicon absorption features were measured for a set of different silicates in both crystalline and amorphous form. The absorption features were implemented in a fitting program (SPEX) in order to model the interstellar dust absorption and scattering. These features, which become more prominent in the spectrum as a function of dust density, are a powerful diagnostics for the denser regions of our Galaxy. The shape and observed energy of these features may indeed reveal the composition and abundance of the dust grains. As a test case we applied our models to a high quality spectrum of a bright background X-ray source located in the vicinity of the Galactic Center.
\end{abstract}

The Life Cycle of Dust in the Universe: Observations, Theory, and Laboratory Experiments - LCDU 2013, 18-22 November 2013

Taipei, Taiwan 


\begin{tabular}{l||l|l|} 
Samples & Chemical formula & Form \\
\hline \hline Olivine & $\mathrm{Mg}_{1.56} \mathrm{Fe}_{0.4} \mathrm{Si}_{0.91} \mathrm{O}_{4}$ & crystal \\
\hline Pyroxene & $\mathrm{Mg}_{0.9} \mathrm{Fe}_{0.1} \mathrm{SiO}_{3}$ & crystal \\
\hline Pyroxene & $\mathrm{Mg}_{0.9} \mathrm{Fe}_{0.1} \mathrm{SiO}_{3}$ & amorphous \\
\hline Enstatite & $\mathrm{MgSiO}_{3}$ & crystal \\
\hline Pyroxene & $\mathrm{Mg}_{0.6} \mathrm{Fe}_{0.4} \mathrm{SiO}_{3}$ & amorphous \\
\hline Pyroxene & $\mathrm{Mg}_{0.6} \mathrm{Fe}_{0.4} \mathrm{SiO}_{3}$ & crystal \\
\hline Hyperstene & $\mathrm{Mg}_{1.502} \mathrm{Fe}_{0.498} \mathrm{Si}_{2} \mathrm{O}_{6}$ & crystal
\end{tabular}

Table 1: Sample compounds

\section{Introduction}

$\mathrm{X}$-rays are an excellent probe for interstellar dust. In the soft X-rays it is possible to measure non-blended absorption features of, for instance, silicon, magnesium, iron and oxygen. The oscillatory modulations near the photoelectric absorption edges of these features may reveal the composition and and abundance of the dust grains. We present newly acquired laboratory data in the X-ray band of several silicate compounds. We focus on the absorption features caused by silicon, i.e. in this case the silicon K-edge absorption feature at $1839 \mathrm{eV}$. The absorption features were implemented in a fitting model (SPEX [U]) in order to model the interstellar dust absorption. As a test case we apply our models to a high quality spectrum of the bright X-ray source GX5-1 located in vicinity of the Galactic Center.

\section{Synchrotron measurements}

The absorption edges of magnesium and silicon of seven samples were measured (see Table $\mathbb{W}$ ). The measurements were done at the synchrotron facility Soleil in Paris, France with the Lucia beam line. Magnesium and silicon absorption features were measured for a set of different silicates in both crystalline and amorphous form. In this proceeding we only show the results of the analysis of the silicon absorption edge.

\section{X-ray absorption fine structure}

X-ray absorption fine structure (XAFS) is the modulation of an atom's X-ray absorption probability due to the chemical and physical state of the atom. When an X-ray photon excites a core electron, the wave function of the outwardly propagating photo-electron will interfere with the waves that scattering from the neighbouring atoms. As can be seen in Figure $\mathbb{W}$ this interference produces an oscillatory fine structure, which is characteristic for the chemical composition of the silicate.

We analysed the differences between the compounds (Zeegers et al. in preparation) and found that each sample produces characteristic absorption features due to the structure and compound of the silicate. It is also possible to distinguish whether the silicates are in crystal or amorphous form. 


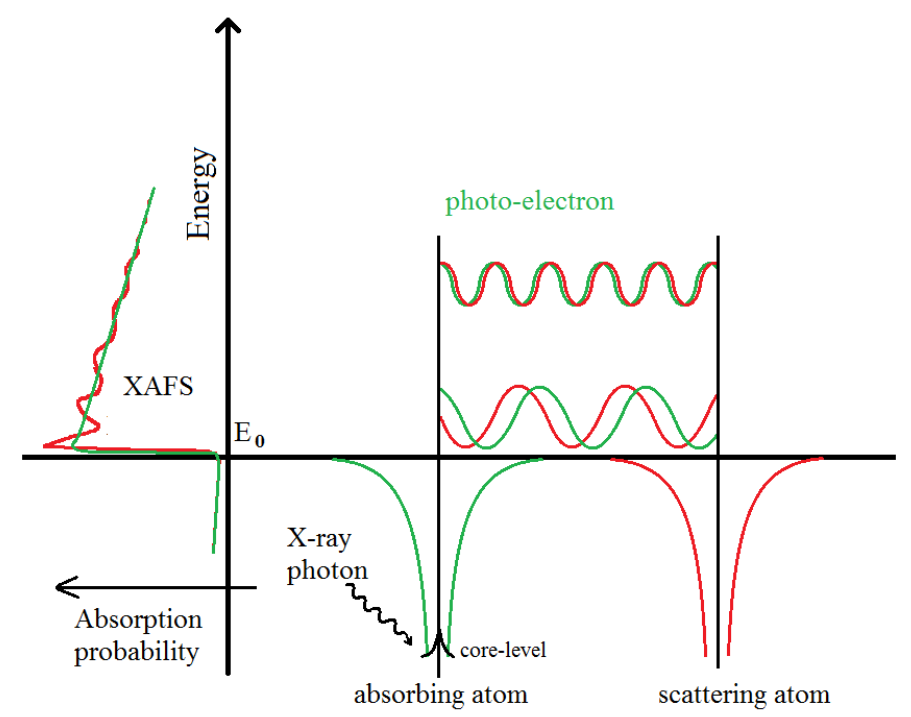

Figure 1: This figure shows a graphical explanation of the XAFS. When an X-ray photon excites a core electron, the wave function of the photo-electron will interfere with the waves that scatter from the neighbouring atom. This interference produces an oscillatory fine structure.

The XAFS features of all the samples were converted to cross sections and added to the fitting model SPEX.

\section{Fit to the spectrum of the X-ray binary GX5-1}

GX5-1 is a low mass X-ray binary at an estimated distance of $8.5 \mathrm{kpc}$ [2]. The spectrum of GX5-1 is used as a test case to apply our models. The X-ray data are taken from the Chandra data archive ([[] $]$ ). In Figure $\square$ we show preliminary results of a fit of the SPEX model of the Si $\mathrm{K}$-edge to the observed to the observed spectrum of GX5-1. The XAFS features of the model in the silicon K-edge are clearly shown. The best fit is shown by the red line. The green line shows the contribution of dust and the blue line the contribution of gas. The SiXIII lines are not produced in the ISM, but by the ionized gas in the source binary.

\section{Results and conclusion}

We analyzed the measurements taken at Soleil and clearly observe a measurable difference in the X-ray absorption fine structure. In Figure $\square$ we show preliminary results of a fit of the SPEX model of the Si-K edge of a silicate to the observed spectrum of GX5-1. From the fit to the spectrum of GX5-1 we can conclude that the silicon abundance is super solar and that the fraction of silicon in dust is about 0.7. In future work we will also add the magnesium absorption profile to SPEX to add further constraints to our model. In this way we will be able to better constrain the data. 


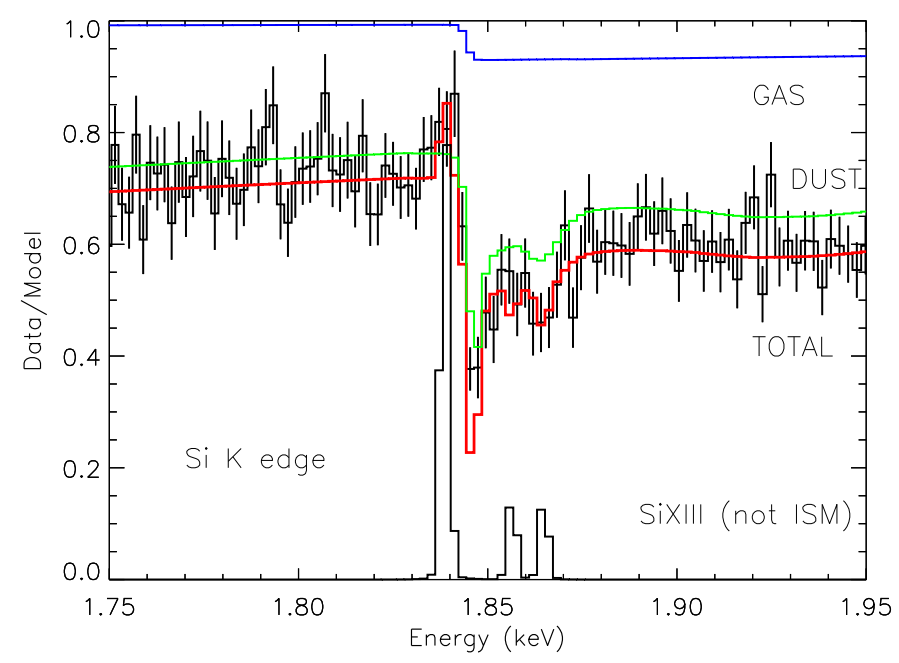

Figure 2: This figure shows the best fit to the spectrum of GX5-1. The spectroscopic data from the ChandraHETG is shown by the black line and the associated error bars. The best fit is shown by the red line. The green line shows the contribution of dust and the blue line the contribution of gas. The SiXIII lines are not produced in the ISM, but by the ionized gas in the source binary.

\section{References}

[1] J. S. Kaastra, R. Mewe, and H. Nieuwenhuijzen. SPEX: a new code for spectral analysis of X \& UV spectra. In K. Yamashita and T. Watanabe, editors, UV and X-ray Spectroscopy of Astrophysical and Laboratory Plasmas, pages 411-414, 1996.

[2] R. K. Smith, T. M. Dame, E. Costantini, and P. Predehl. The X-Ray Halo of GX 5-1. ApJ, 648:452-460, September 2006.

[3] D. P. Huenemoerder, A. Mitschang, D. Dewey, M. A. Nowak, N. S. Schulz, J. S. Nichols, J. E. Davis, J. C. Houck, H. L. Marshall, M. S. Noble, D. Morgan, and C. R. Canizares. TGCat: The Chandra Transmission Grating Data Catalog and Archive. AJ , 141:129, April 2011. 